\title{
Mordedura por Philodryas chamissonis. Presentación de un caso y revisión de la literatura
}

\author{
Patricia Neira O., Leonor Jofré M., David Oschilewski L., Benjamín Subercaseaux S. y Nelson Muñoz S.
}

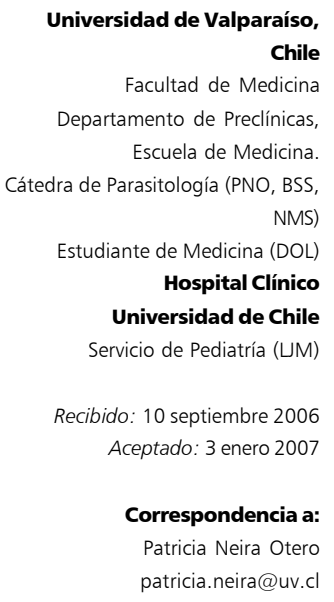

\section{Snake bite by Philodryas chamissonis: a case presentation and literature review}

There are two species of snakes associated with snake bite poisoning in Chile: Philodryas chamissonis and Tachymenis peruviana. A case associated with a $P$. chamissonis bite occuring during a summer activity in San Antonio, V Region, is presented. The bite compromised the dorsum of the right hand between the thumb and the index finger and was initially painless. During the following 24 hours equimotic edema developed up to the shoulder and pectoral region, with intense pain, headache, nausea, fever and appearance of a serohematic bulla on the elbow fold. The patient was treated with antihistamins, systemic steroids, analgesia and antibiotics for 7 days. Other cases of snake bites published in Chile are reviewed and treatment and prevention strategies are proposed.

Key words: snake bite, snake venom; Philodryas chamissonis; Tachymenis peruviana.

Palabras claves: mordedura de culebra; ofidismo; empozoñamiento; Philodryas chamissonis; Tachymenis peruviana.

\section{Introducción}

A las serpientes se les reconoce por la ubicación de los dientes inoculadores y sus características morfológicas. Las serpientes consideradas venenosas son aquellas con dentición proteroglifa (del griego protero $=$ anterior y glifis $=$ muesca), que tienen los colmillos ubicados anteriormente, de tal manera que al clavar la presa, permite que el veneno fluya libremente ${ }^{1} \mathrm{y}$ las solenoglifa (del griego solenius $=$ conductor y glifis = ranura), que es la forma más perfeccionada de evolución del aparato ponzoñoso. A este último grupo pertenecen la serpiente cascabel y víboras entre otras.

Las aglifas (del griego $a=\sin$ y glifis $=$ muesca), poseen dientes maxilares pero sin surco, por lo que el veneno fluye junto con la saliva, lo que atenúa sus efectos. Las opistoglifas (del griego opisto $=$ posterior y glifis $=$ muesca $)$ presentan dientes inoculadores de ubicación posterior, que corresponde a un grado inicial en la especialización del aparato ponzoñoso, a este grupo pertenecen varios miembros de la familia Colubridae ${ }^{1}$.

Los accidentes por serpientes venenosas en América Latina y Centroamérica son, en general, subnotificados; se estima que se producen unos 150.000 accidentes al año, de los cuales $\sim 65 \%$ son hospitalizados. Brasil, Ecuador y Venezuela son los países más afectados, con una incidencia variable de 45 a 1.000 casos por 100.000 habitantes. La mayoría son producidos por serpientes del género Bothrops (jararaca), Crotalus (serpiente cascabel) y con menor frecuencia Lachesis (sururucu) y Microcurus (coral) ${ }^{2}$.

En Chile continental los ofidios están representados por siete formas de la familia Colubridae. Dos tipos de culebras se han relacionado a accidentes por mordeduras en humanos: Philodryas chamissonis (anteriormente Dromicus chamissonis), llamada culebra de cola larga y Tachymenis peruviana, o culebra de cola corta. Philodryas chamissonis se encuentra distribuida entre la III $^{\mathrm{a}}$ y $\mathrm{X}^{\mathrm{a}}$ Región (Atacama a Los Lagos) y $T$. peruviana desde la IV $^{\mathrm{a}}$ a la $\mathrm{IX}^{\mathrm{a}}$ Región. Ambas tienen dentición opistoglifa ${ }^{3}$.

Las culebras descritas en nuestro país atacan al hombre sólo en defensa propia. Los accidentes por mordeduras se presentan esporádicamente y se ubican en las extremidades y en los dedos de personas que toman estas culebras ${ }^{4,5}$.

Philodryas chamissonis es el ofidio de mayor tamaño en nuestro país, puede tener hasta $2 \mathrm{~m}$ de longitud. Se caracteriza por presentar en el dorso una línea longitudinal central y continua de color pardo oscuro casi negra, con franjas amarillas laterales, que permite su identificación morfológica. Destacan sus ojos con dos escamas post oculares y las pupilas circulares.

Tachymenis peruviana mide de 40 a $60 \mathrm{~cm}$, posee 
una cola corta, colores menos vistosos y tiene una franja central blanquecina y dos laterales más oscuras. Las pupilas están dispuestas en forma vertical ${ }^{6}$.

Presentamos el caso de una paciente mordida por $P$. chamissonis, se discuten las manifestaciones clínicas, el tratamiento y prevención de estos accidentes.

\section{Caso Clínico}

Adolescente de sexo femenino, 14 años de edad. Durante un campamento de verano en carpa junto a su familia, en la localidad del Quisco, V $V^{\mathrm{a}}$ Región, se agachó a recoger una pelota bajo unos matorrales. En ese momento sintió que algo apretaba su mano izquierda. Al retirar la mano vio una gran culebra prendada a la zona del dorso de la mano, entre los dedos pulgar e índice (Figura 1). Ella cogió la cabeza de la culebra por detrás, con la mano libre y al cabo de algunos segundos el reptil soltó la mano, alcanzando a enrollarse alrededor del brazo izquierdo. Para evitar que la culebra escapara, los padres la mataron y guardaron en un recipiente.

La culebra medía $137 \mathrm{~cm}$ de longitud, siendo su cola de $35 \mathrm{~cm}$. En la zona dorsal presentaba una franja oscura al centro flanqueada por franjas claras laterales (Figura 2). No se logró encontrar los dientes inoculadores posteriores.

La mordedura no provocó dolor al momento del accidente y no se logró apreciar el sitio de inoculación. Aproximadamente al cabo de 30 minutos, inició un intenso dolor y aumento de volumen en el sitio de la mordedura (Figura 3).

Fue llevada al servicio de urgencia del hospital Carlos van Buren, Valparaíso, V $V^{\mathrm{a}}$ Región. En la evaluación inicial se encontró un edema circunferencial que abarcaba hasta la unión del tercio medio inferior del brazo, con una herida puntiforme en la mano izquierda y erosión de la zona vecina, los dedos de la extremidad afectada se encontraban fríos, no refería mialgias.

Fue hospitalizada con el diagnóstico de mordedura por ofidio y observación de un síndrome compartamental. Se indicó como tratamiento corticosteroides y anti-histamínicos endovenosos, analgésicos tipo AINE y ampicilina parenteral. Las medidas locales indicadas fueron brazo en alto y aplicación de hielo.

De los exámenes de laboratorio solicitados destacaban: protrombinemia: 57\% (VN=70-100\%), TTPK: 41 seg $(\mathrm{VN}=26-36 \mathrm{seg})$, recuento de plaquetas: 300.000 $\left(\mathrm{VN}=150.000-400.000 / \mathrm{mm}^{3}\right)$, hematocrito: $44 \%(\mathrm{VN}=$ 34-44\%); hemoglobinemia: 14,6 gr/dL (VN=11,6-15 gr/ dL). No se solicitó recuento de leucocitos.

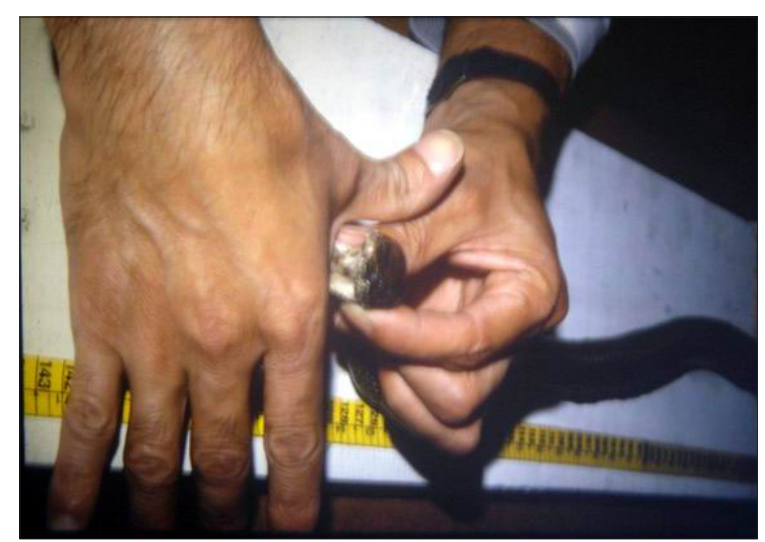

Figura 1. Representación de la escena del accidente.

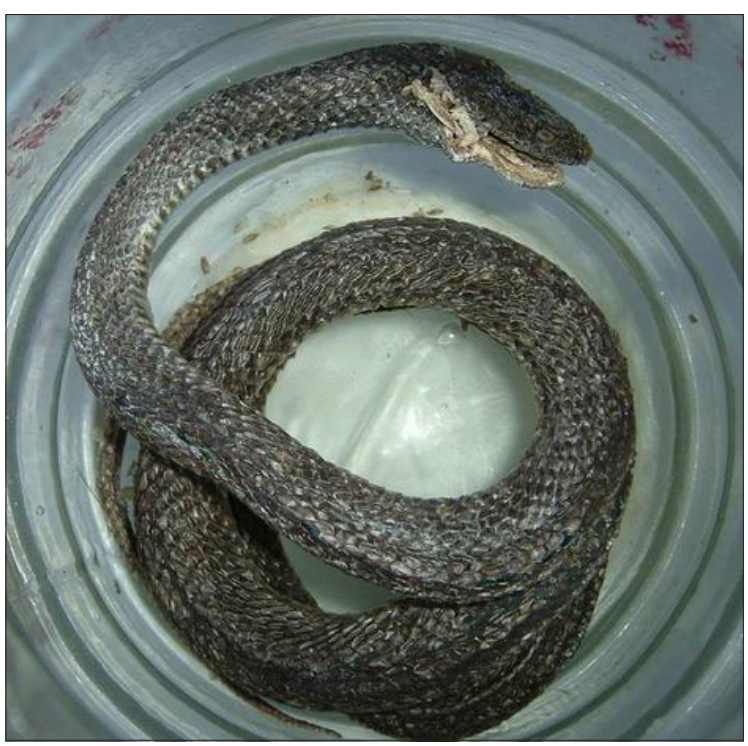

Figura 2. Philodryas chamissonis muerta, en un recipiente.

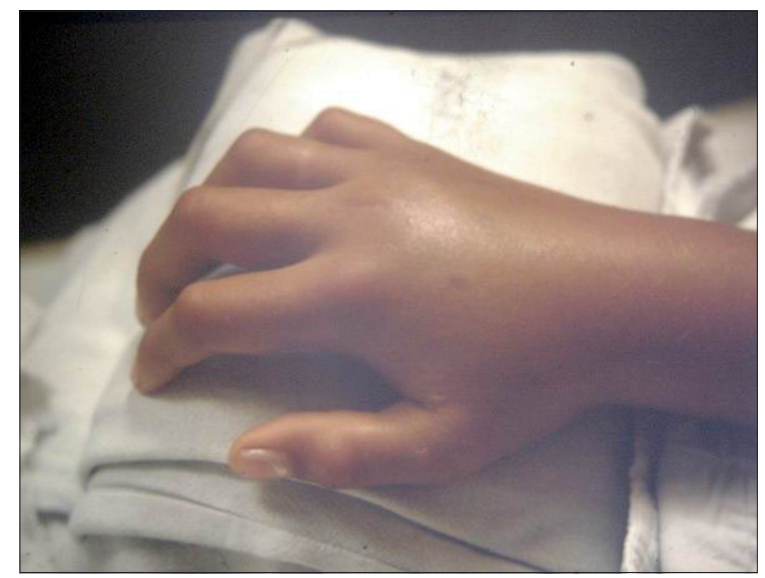

Figura 3. Edema de la mano a las 24 horas de evolución. 
Figura 4. Progresión del edema a las 48 horas de evolución.

Figura 5. Edema acartonado de la mano.
Evolucionó en regulares condiciones generales las primeras 24 horas, sin fiebre, pero con un intenso dolor en la extremidad superior izquierda. El edema se extendió hasta la región del hombro y zona pectoral y los dedos tomaron un aspecto equimótico (Figura 4). Destacó en el examen el dolor a la palpación de la zona afectada y la consistencia acartonada del edema (Figura 5). Presentó cefalea intensa, náuseas y febrículas, sin evidencias de sangramientos. La orina fue evaluada diariamente por colorometría a ojo desnudo, para la detección de hemoglobinuria.

Se mantuvo el tratamiento con analgésicos y corticosteroides endovenosos y el edema del antebrazo y brazo comenzó a disminuir. A las 48 horas de evolución aparecieron bulas serohemorrágicas en la zona del pliegue del codo derecho, disminuyó la cefalea, desaparecieron las náuseas y la fiebre, con persistencia del dolor en la extremidad. La movilidad de los
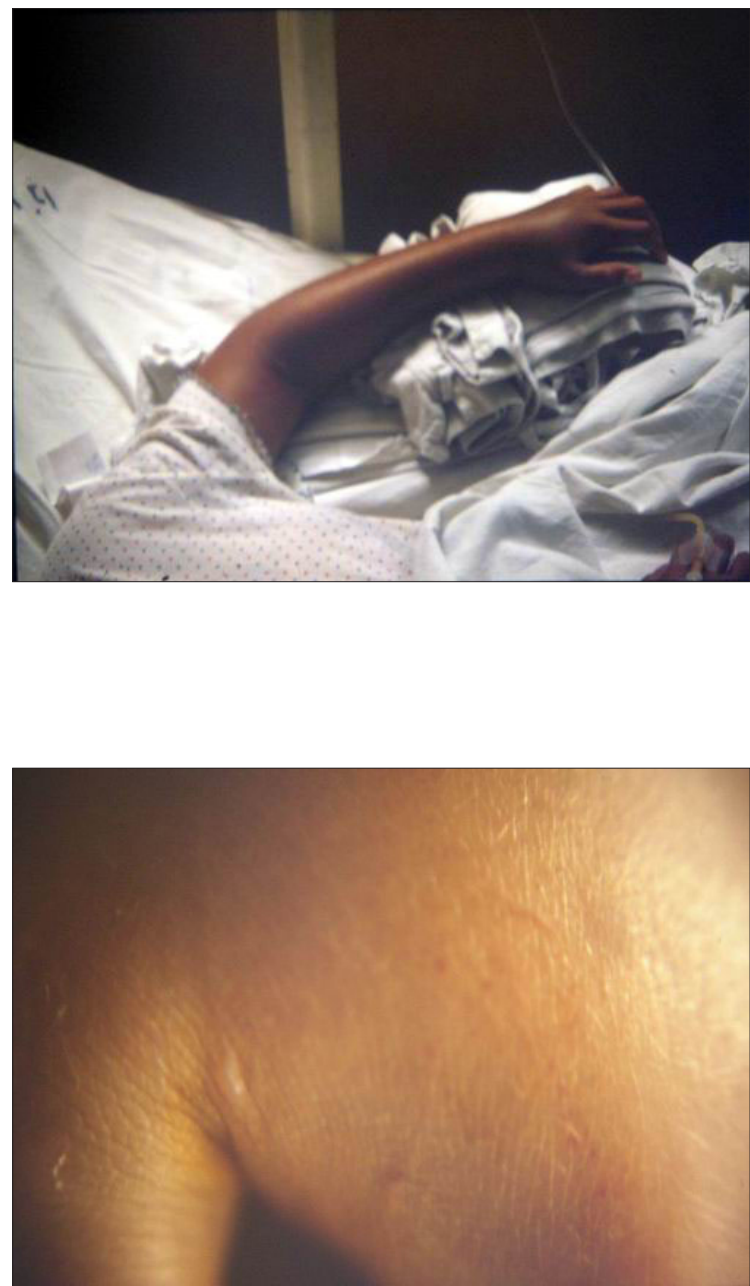

dedos y la muñeca estaban limitadas, pero con palpación de pulsos arteriales normales. Los cirujanos descartaron un síndrome compartamental.

A las 72 horas de evolución, tanto el edema como el dolor se encontraban en franca regresión y se suspendieron los corticosteroides y anti-histamínicos. Al cuarto día de evolución se dio de alta, casi sin molestias, con indicación de completar tratamiento con prednisona durante dos días y el tratamiento antimicrobiano por seis días.

\section{Discusión}

Las primeras contribuciones a la herpetología chilena se remontan al siglo XVIII, y recién en el siglo XX aparecen los primeros registros de accidentes por culebras en la literatura médica de nuestro país. El primer relato se realizó en una sesión de la Sociedad de Cirugía de Chile el $1^{\circ}$ de junio de 1938 , donde se presentaron dos casos ocurridos en el año 1932, comentados por el Dr. Costa ${ }^{6}$. La importancia del tema, en aquella época, permitió abrir un nuevo capítulo en la patología nacional; sin embargo, a la fecha, son infrecuentes los accidentes por mordedura de culebras (Tabla 1).

Dos especies de culebras causan accidentes en Chile: $P$. chamissonis y $T$. peruviana. El mayor número de accidentes registrado es por $T$. peruviana ${ }^{3}$. Las culebras son predadoras activas, capturan sus presas y las enrollan violentamente para su ingestión posterior. Tienen dientes ubicados en la parte anterior de las arcadas mandibulares y dos dientes inoculadores en la región posterior del maxilar superior, que están recubiertos por una vaina envolvente y protectora, con un surco en su cara anterior que se extiende desde la base a las proximidades del ápice. Durante la mordedura estos dientes se proyectan, siendo necesario que realicen una amplia apertura bucal.

El veneno extraído es de consistencia lechosa, viscosa, con granulaciones. La cantidad producida es variable, alcanza a $20 \mathrm{mg}$, y se reproduce una vez exprimido. Las temperaturas altas lo destruyen y las frías lo mantienen. La acción de la ponzoña es proteolítica $^{9,10}$, lo que explica el edema y el dolor, con una acción hemolítica poco marcada. En algunos casos de mordedura por $P$. chamissonis, se ha reportado hipo-protrombinemia, que plantea la existencia de un compuesto del tipo cumarínico en el veneno de esta culebra $^{7-9}$.

El veneno inyectado en ratones en forma experimental, produce intranquilidad e hiperestesia en el punto de inoculación, temblor, ataxia y parálisis del tren posterior, que puede llevar a la muerte en un plazo de 30 a 115 minutos. Una situación similar ocurre en 


\section{Especie}

No identificada ${ }^{19}$

Año

1932

\section{Sitio mordedura}

Primer espacio interdigital de la mano izquierda

\begin{tabular}{|c|c|c|c|}
\hline T. peruviana ${ }^{19}$ & 1932 & $\mathrm{NC}$ & Masculino \\
\hline No identificada ${ }^{6}$ & 1934 & Dorso de la mano & Masculino \\
\hline $\begin{array}{l}\text { T. peruviana } \\
\text { según Donoso } \\
\text { Barros }^{6}\end{array}$ & 1938 & Dedo índice & Masculino, naturalista \\
\hline $\begin{array}{l}\text { No identificada, } \\
\text { (Por tamaño }\end{array}$ & 1938 & $\begin{array}{l}\text { Mano derecha } 2^{\circ} \text { metacarpiano, } \\
\text { borde externo }\end{array}$ & Masculino, 43 a, carpintero \\
\hline
\end{tabular}

$30 \mathrm{~cm}$,

T. peruviana) $)^{19}$

\section{Sexo, edad, actividad}

Masculino, 31 a, campesino

\section{Clínica}

Erosiones en la base del pulgar izquierdo, con gran edema que abarcó hasta el tórax, con extensa equimosis Flictenas en el dorso de la mano

Se relata como "idéntico al anterior"

Edema de extremidad superior y hemitórax derecho

Sensación de clavada de alfiler al momento de la mordedura. Edema pronunciado, duro y blanco. Dolor axilar, infarto ganglionar. Mareos

Heridas puntiformes en el borde externo de la segunda articulación metacarpo falángica. Edema del miembro superior derecho indoloro, con manchas equimóticas dispersas en la mano, antebrazo y cara interna del brazo. Mancha equimótica de $10 \mathrm{~cm}$ de diámetro en la axila, acompañada de edema. El dolor aparece a las $22 \mathrm{~h}$, con aumento de edema y equimosis, que alcanza a la región glútea transversal a columna

$\begin{array}{lcll}\text { P. } \text { chamissonis }^{20} & \text { NC } & \text { NC } & \text { NC } \\ \text { T. peruviana }{ }^{12} & \text { NC } & \text { NC } & \text { NC } \\ T \text {. peruviana } & 1946 & \begin{array}{l}2^{\text {a }} \text { articulación falángica dedo medio } \\ \text { de la mano izquierda }\end{array} & \text { Masculino, } 18 \text { a } \\ T . \text { peruviana }^{12} & 1949 & \begin{array}{l}\text { Cara dorsal de la primera falange } \\ \text { dedo medio, mano derecha }\end{array} & \text { Masculino, } 40 \text { a, jardinero }\end{array}$
$\mathrm{NC}$ $\mathrm{NC}$

Pequeñas heridas y hemorragia moderada. Dolor y edema progresivo que alcanzó la parte alta del brazo. Infarto de ganglios epitrocleares y de la axila izquierda Dolor y ardor en el momento de la mordedura. Pequeñas manchas rojizas en la cara dorsal de la primera falange del dedo medio. Edema del dedo, mano y antebrazo; frío, indoloro y pálido. Cefalea y astenia

T. peruviana $^{12} \quad 1951$ Falange media del dedo meñique Masculino, 18 a, obrero parte externa mano izquierda

Dolor y sudoración en el momento de la mordedura. Cuatro manchas negras ( 2 en el extremo distal y 2 en el extremo proximal del dedo) Edema progresivo y equimosis Ganglios supra-epitrocleares y axilares con aumento de volumen y dolorosos

P. chamissonis ${ }^{8} \quad 1954 \quad$ Raíz del índice derecho

Masculino, 25 a

Intenso dolor punzante, edema del dedo y mano. Mareos, adinamia y cefalea. Mancha equimótica en la cara interna del brazo y hueco axilar derechos. Adenopatías epitroclear y axilar

$\begin{array}{llll}\text { T. } \text { peruviana }^{12} & 1956 & 1^{\text {a }} \text { falange anular derecho } & \text { Masculino, } 36 \text { a, carabinero } \\ \text { T. } \text { peruviana }^{12} & \text { NC } & \begin{array}{l}\text { Falange proximal índice mano } \\ \text { izquierda, borde súpero interno }\end{array} & \text { Masculino, } 30 \text { a, mecánico }\end{array}$

Dolor, sensación de compresión local, edema progresivo de dedos hasta axila. Equimosis en el dorso de la mano y muñeca. Ganglios sin aumento de volumen

Mordedura poco dolorosa. Edema progresivo de dedo al brazo. Dolor en articulaciones, ganglios supraepitrocleares con aumento de volumen, sin reacción ganglionar axilar

\begin{tabular}{|c|c|c|c|c|}
\hline T. peruviana ${ }^{12}$ & 1958 & Mano izquierda & Masculino, 42 a & $\begin{array}{l}\text { Dolor poco acentuado y edema de la extremidad } \\
\text { superior izquierda que abarcó a hemitórax izquierdo. } \\
\text { Equimosis en la zona escapular }\end{array}$ \\
\hline P. chamissonis ${ }^{10}$ & 1960 & NC & NC & "Síntomas alarmantes" \\
\hline T. peruviana ${ }^{10}$ & 1961 & NC & $\mathrm{NC}$ & NC \\
\hline P. chamissonis ${ }^{11}$ & 1993 & Dedo índice derecho & Masculino, 17a & $\begin{array}{l}\text { Herida puntiforme en la cara posterior de la falange } \\
\text { distal de dedo índice. Intenso dolor, edema y } \\
\text { equimosis de la extremidad superior derecha }\end{array}$ \\
\hline P. chamissonis ${ }^{11}$ & 1993 & Pliegue del codo derecho & Masculino, $11 \mathrm{a}$ & $\begin{array}{l}\text { Herida punzante en el pliegue del codo. Edema duro, } \\
\text { gran hematoma indoloro de superficie extensora del } \\
\text { antebrazo }\end{array}$ \\
\hline
\end{tabular}

$\mathrm{NC}=$ no consignado 
anuros y lagartijas 9

En humanos estos accidentes ocurren en personas jóvenes, de sexo masculino, que han estado jugando con la culebra y se ubican, de preferencia, en los dedos. En la zona de inoculación aparece un halo hemorrágico, y al cabo de 24 horas aparece edema de la extremidad afectada, de tipo equimótico. La extensión de la lesión va a depender de la cantidad de veneno inoculada.

Si el accidente es producido por $P$. chamisonnis, el edema es caliente, rubicundo e hipersensible, con aparición de flictenas y manchas equimóticas; que puede acompañarse de fiebre e incluso hipertermia ${ }^{10}$. En mordeduras por $T$. peruviana, en cambio, el edema es blanco, frío, la mayoría de las veces indoloro, con sensibilidad de las zonas ganglionares comprometidas secundario a infartos. En el hemograma puede encontrarse leucocitosis y desviación izquierda ${ }^{3,4}$. La sintomatología tiende a disminuir en cuatro a siete días, no provoca mortalidad y no existe un antídoto específico para las mordeduras ocasionadas por estas culebras ${ }^{10,11}$.

Las culebras, al ser atacadas se defienden y muerden con los dientes anteriores o por un rato corto, por lo que la ponzoña no alcanza a penetrar la piel o puede no disponer de veneno en ese momento. La víctima, al tratar de defenderse, puede fracturar o desprender los dientes, quedando incluidos en la piel y dejan como manifestación una herida sangrante ${ }^{12}$. En Chile, hay 18 accidentes descritos (Tabla 1), en su mayoría afectaron a hombres jóvenes y adultos; dos casos corresponden a pacientes pediátricos.

El tratamiento es sintomático, no se debe realizar torniquetes ni succión de la zona de la mordedura ${ }^{13}$. El uso de corticosteroides se ha asociado a disminución del edema en experiencias nacionales ${ }^{10}$. En estos casos se debe revisar la inmunización anti-tetánica del paciente. No está indicado el uso de antimicrobianos

\section{Tabla 2. Recomendaciones para el tratamiento de las mordeduras}

\section{por culebras en Chile}

- Inmovilizar la extremidad afectada

- No usar torniquete ni succionar la herida

- Aseo local

- Revisar el estado de inmunización anti-tetánica

- Hospitalizar para hidratación y analgesia

- Uso de corticosteroides sistémicos

- Antimicrobianos sólo en caso de sospecha de infección: amoxicilina/ácido clavulánico + ciprofloxacina

- Suero específico no disponible y no indicado

- Evaluación quirúrgica y debridamiento en caso necesario. Descartar un síndrome compartamental en forma profiláctica, dado que las complicaciones infecciosas son poco frecuentes ${ }^{14}$. Se justifica el uso de antimicrobianos cuando hay sospecha de infección y compromiso de tejidos blandos ${ }^{14,15}$. A partir de secreciones obtenidas de abscesos ocasionados por mordeduras de serpientes, se han aislado cocáceas grampositivas y bacilos gramnegativos como Escherichia coli y Morganella morgagni ${ }^{16}$. La complicación más grave en estos casos es la fasceítis necrosante por Aeromona hydrophila ${ }^{17}$, en casos menos frecuentes por Vibrio vulnificus ${ }^{18}$; en los pacientes descritos en Chile no se hace alusión a complicaciones infecciosas. En caso de sospecharse una infección, se recomienda el uso de amoxicilina con ácido clavulánico más ciprofloxacina, para alcanzar cobertura de agentes grampositivos y bacilos gramnegativos. La conducta a seguir frente a una mordedura de culebra se resume en la Tabla 2.

En relación con la prevención, se debe evitar jugar o molestar a estas culebras y tener precaución al realizar actividades en zonas campestres.

\section{Conclusiones}

Los accidentes por mordedura de culebras son poco frecuente en Chile. Hay dos géneros de culebras asociadas a estos accidentes: $P$. chamissonis y $T$. peruviana, con dentición opistoglifa, con dientes ubicados en su parte posterior, que eventualmente pueden causar emponzoñamiento ${ }^{7,8}$. Los casos reportados en Chile presentan edema progresivo, de tipo equimótico, doloroso, y con manifestaciones sistémicas como cefalea y fiebre en el caso de $P$. chamisonnis.

El tratamiento se realiza con corticosteroides endovenosos y manejo del dolor. Se debe revisar la inmunización anti-tetánica de los accidentados. Los antimicrobianos no están indicados de rutina. En caso de sospecha de infección, se debe dejar tratamiento con cobertura para cocáceas grampositivas y bacilos gramnegativos. En relación con la prevención, hay que tomar precauciones en las actividades realizadas en ambientes rurales o al aire libre, ya que la mayoría de las mordeduras ocasionadas por estas culebras son en defensa propia.

\section{Resumen}

El ofidismo es el accidente provocado por la mordedura de una serpiente. En Chile se encuentran 2 tipos de culebras capaces de provocar emponzoñamiento: Philodryas chamissonis y Tachymenis peruviana. Se presenta el caso de una mordedura por $P$. chamissonis, 
ocurrida durante una actividad veraniega en la comuna de San Antonio, V Región. La mordedura comprometió la zona del dorso de la mano entre los dedos pulgar e índice de la extremidad superior izquierda, inicialmente indolora. Evolucionó las primeras 24 horas con edema equimótico que abarcó hasta la zona del hombro y pectoral, con dolor intenso, cefalea, náu- seas, fiebre y posterior aparición de bulas serohemorrágicas en el pliegue del codo. La paciente se trató con antihistamínicos, corticoides sistémicos, analgesia y antimicrobianos por 7 días. Se discuten los accidentes por mordedura de culebras publicados en la literatura nacional, así como su tratamiento y las medidas de prevención.

\section{Referencias}

1.- Espinoza R. En relación a Cleopatra y los venenos de serpiente. Rev Méd Chile 2001; 129: $1222-6$

2.- Pereira I D. Ofidismo. Rev Ass Med Brasil 2001; 47: 24-91.

3.- Donoso Barros R. Reptiles de Chile. Ediciones de Universidad de Chile, Santiago, Chile, 1966.

4.- Schenone H, Reyes H. Animales ponzoñosos de Chile. Bol Chil Parasitol 1965; 20: 104-9.

5.- Núñez H. Culebras chilenas: más allá del mito. Naturaleza 1984; 2: 16-9.

6.- Johow A. Mordedura de culebra. Bol Soc Cirugía (Rev Méd Chile) 1938; 66: 661-3.

7.- Gajardo- Tobar R. ¿Los ofidios chilenos son capaces de envenenar?. Bol Hosp Viña del Mar (Chile) 1947; 3: 43-51.

8.- Schenone H, Bertín V, Mann G. Un nuevo caso de ofidismo. Bol Chil Parasitol 1954; 9:
88-9.

9.- Donoso-Barros R, Cárdenas S. Estudio del veneno de Dromycus chamissonis (Wiegman). Inv Zool Chilenas 1959; 5: 935

10.- Donoso Barros R. Emponzoñamiento por ofidios chilenos. Rev Pediatr Clin Soc 1961; 1: 65-79.

11.- Arzola J, Schenone H. Dos casos nuevos de ofidismo en Chile. Bol Chil Parasitol 1994; 49: $69-70$

12.- Gajardo-Tobar R. Cinco casos de ofidismo. Bol Hosp Viña del Mar (Chile) 1958; 15: 2538.

13.- Gold B S, Dart R C, Barish R A. Bites of venomous snakes. N Engl J Med 2002; 347: 347-56.

14.- Juckett G, Hancox J G. Venomous snakebites in the United States: management review and update. Am Fam Phys 2002; 65: 1367-74.

15.- Tagweyi D, Ball D E, Nhachi C F. Routine prophylactic antibiotic use in the management of the snakebite. BMC Clinical Pharmacol 2001; 1: 4.

16.- Blaylock R S. Antibiotic use and infection in snake bite victim. S Afr Med J 1999; 89: 874-6.

17.- Angel M F, Zhang F, Jones M, Henderson J, Chapman S. Necrotizing fasciitis of the upper extremity resulting from a water moccasin bite. South Med J 2002; 95: 10904.

18.- Wu C H, Hu W H, Hung D Z, Peng Y C, Yang D Y. Snakebite complicated with Vibrio vulnificus. Emerg Med J 2002; 19: 142.

19.- Rayo F, Covarrubias R, Ruiz M. Mordedura de serpiente. Bol Soc Cirugía (Rev Méd Chile) 1938; 66: 773-9.

20.- Gigoux E R. Los ofidios chilenos. Santiago de Chile. Bol Museo Nacional Hist Natural 1940; 18: 5 . 